\title{
Analysis of periodic dislocation networks using x-ray diffraction and extended finite element modeling
}

\author{
E. Wintersberger ${ }^{1, a)}$ N. Hrauda, ${ }^{1}$ D. Kriegner, ${ }^{1}$ M. Keplinger, ${ }^{1}$ G. Springholz, ${ }^{1}$ J. Stangl, ${ }^{1}$ \\ G. Bauer, ${ }^{1}$ J. Oswald, ${ }^{2}$ T. Belytschko, ${ }^{2}$ C. Deiter, ${ }^{3}$ F. Bertram, ${ }^{3}$ and O. H. Seeck ${ }^{3}$ \\ ${ }_{1}^{1}$ Department of Semiconductor Physics, University of Linz, A-4040 Linz, Austria \\ ${ }^{2}$ Theoretical and Applied Mechanics, Northwestern University, 2145 Sheridan Road, Evanston, \\ Illinois 60208-3111, USA \\ ${ }^{3}$ HASYLAB, DESY, Notkestrasse 85, D-22607 Hamburg, Germany
}

(Received 19 February 2010; accepted 15 March 2010; published online 1 April 2010)

\begin{abstract}
We combine the extended finite element method with simulations of diffracted x-ray intensities to investigate the diffusely scattered intensity due to dislocations. As a model system a thin $\mathrm{PbSe}$ epitaxial layer grown on top of a PbTe buffer on a CdTe substrate was chosen. The PbSe film shows a periodic dislocation network where the dislocations run along the orthogonal $\langle 110\rangle$ directions. The array of dislocations within this layer can be described by a short range order model with a narrow distribution. (C) 2010 American Institute of Physics. [doi:10.1063/1.3379298]
\end{abstract}

Heteroepitaxial semiconductor systems, formed by layers of materials with different bulk lattice parameters deposited on a substrate, play a crucial role in semiconductor devices. ${ }^{1}$ The strain induced by the lattice mismatch between the different layers can be used to enhance the mobility of charge carriers in electrically active regions. ${ }^{2}$ However, once a particular layer exceeds a certain critical thickness plastic relaxation takes place by the formation of dislocations. ${ }^{3}$ The dislocations are usually stochastically distributed and therefore may produce an inhomogeneous strain field. Furthermore, dislocation threading segments penetrate the layer stack and act as scattering centers in electrically active regions and hence may decrease the carrier mobility. Therefore, the characterization of dislocations in such systems plays an important role in process optimization. Unlike transmission electron microscopy or scanning tunneling microscopy (STM), x-ray diffraction (XRD) provides information from a large region of the sample and is therefore a well suited tool for this purpose. The diffusely scattered intensity due to dislocations has been studied by many groups. ${ }^{4-9}$ Most of these works use analytical formulas for the calculation of the displacement field due to the dislocations, which are limited to isotropic materials and simple dislocation geometries. In this work, an approach based on the finite element method (FEM) was used to overcome these limitations.

In a previous study ${ }^{10}$ dislocation networks in compressively strained $\mathrm{PbTe}$ films grown on $\mathrm{PbSe}$ substrates were investigated by means of STM. It was found that the dislocations form a periodic network with a mean distance of 10 $\mathrm{nm}$ in the PbTe film. From a device point of view, periodic dislocation networks are of particular interest. Although current dislocation distances are still too small (see below), this effect might be used to create dislocation free regions for devices. For devices both tensile as well as compressively strained layers are required. According to Ref. 11, the relaxation behavior of an epilayer depends not only on the magnitude but also on the sign of the strain. Therefore, we investigated here a tensile strained $\mathrm{PbSe}$ film grown on top of a

${ }^{a)}$ Electronic mail: eugen.wintersberger@jku.at.
$\mathrm{PbTe}$ buffer layer, to compare it to the opposite case of Ref. 10.

The sample (VA696a) was grown on a CdTe substrate using molecular beam epitaxy. First, a $100 \mathrm{~nm}$ thick PbTe buffer layer was deposited followed by a 20 monolayers (6.1 $\mathrm{nm})$ thick PbSe layer. Both layers were grown at a temperature of $360{ }^{\circ} \mathrm{C}$. The relevant parameters of all involved materials are shown in Table I. The lattice mismatch between the $\mathrm{PbSe}$ and $\mathrm{PbTe}$ layers is defined as

$$
\epsilon=\frac{a_{\mathrm{PbSe} \text { bulk }}-a_{\mathrm{PbTe} \|}}{a_{\mathrm{PbTe} \|}},
$$

with $a_{\mathrm{PbTel}}$ denoting the in-plane lattice parameter of the PbTe buffer.

XRD experiments were performed at beamline P08 at the PETRA III synchrotron at HASYLAB in Hamburg, Germany. Reciprocal space maps (RSMs) around the (002) and (115) CdTe Bragg peaks were recorded at a beam energy of $8944 \mathrm{eV}$. The measurements are shown in Figs. 1(b) and 1(c). From the (115) RSM it follows that the PbTe buffer is grown pseudomorphically on the CdTe substrate. Therefore, the lattice mismatch between the buffer and the PbSe layer takes a value of $\epsilon=-0.055$. From the position of the PbSe peak in the (115) RSM an in-plane tensile strain of the PbSe layer with respect to the buffer of $\epsilon_{\|, s}=-0.044$ was obtained. This corresponds to a degree of relaxation of $R=\left(a_{\mathrm{PbSe}, \|}\right.$ $\left.-a_{\mathrm{PbTe}, \|}\right) /\left(a_{\mathrm{PbSe}, \text { bulk }}-a_{\mathrm{PbTe}, \|}\right)=0.81$. Clearly the PbSe layer has undergone plastic relaxation. The absolute value of the Burgers vector of the dislocations in this particular case is given by $|\mathbf{b}|=a_{\mathrm{PbTel}} / \sqrt{2}=4.58 \AA$ and their mean distance can be calculated as $\langle L\rangle_{\text {strain }}=|\mathbf{b}| / \epsilon_{\|, s}=10.4 \mathrm{~nm}$.

TABLE I. Lattice parameters and elastic constants of the materials involved in the simulations.

\begin{tabular}{lcccc}
\hline \hline & $\begin{array}{c}a \\
(\AA)\end{array}$ & $\begin{array}{c}c_{11} \\
\left(\mathrm{~N} / \mathrm{m}^{2}\right)\end{array}$ & $\begin{array}{c}c_{12} \\
\left(\mathrm{~N} / \mathrm{m}^{2}\right)\end{array}$ & $\begin{array}{c}c_{44} \\
\left(\mathrm{~N} / \mathrm{m}^{2}\right)\end{array}$ \\
\hline $\mathrm{CdTe}$ & 6.482 & $5.35 \times 10^{10}$ & $3.67 \times 10^{10}$ & $1.99 \times 10^{10}$ \\
$\mathrm{PbTe}$ & 6.462 & $9.36 \times 10^{10}$ & $0.67 \times 10^{10}$ & $1.63 \times 10^{10}$ \\
$\mathrm{PbSe}$ & 6.126 & $12.37 \times 10^{10}$ & $1.93 \times 10^{10}$ & $1.59 \times 10^{10}$ \\
\hline \hline
\end{tabular}




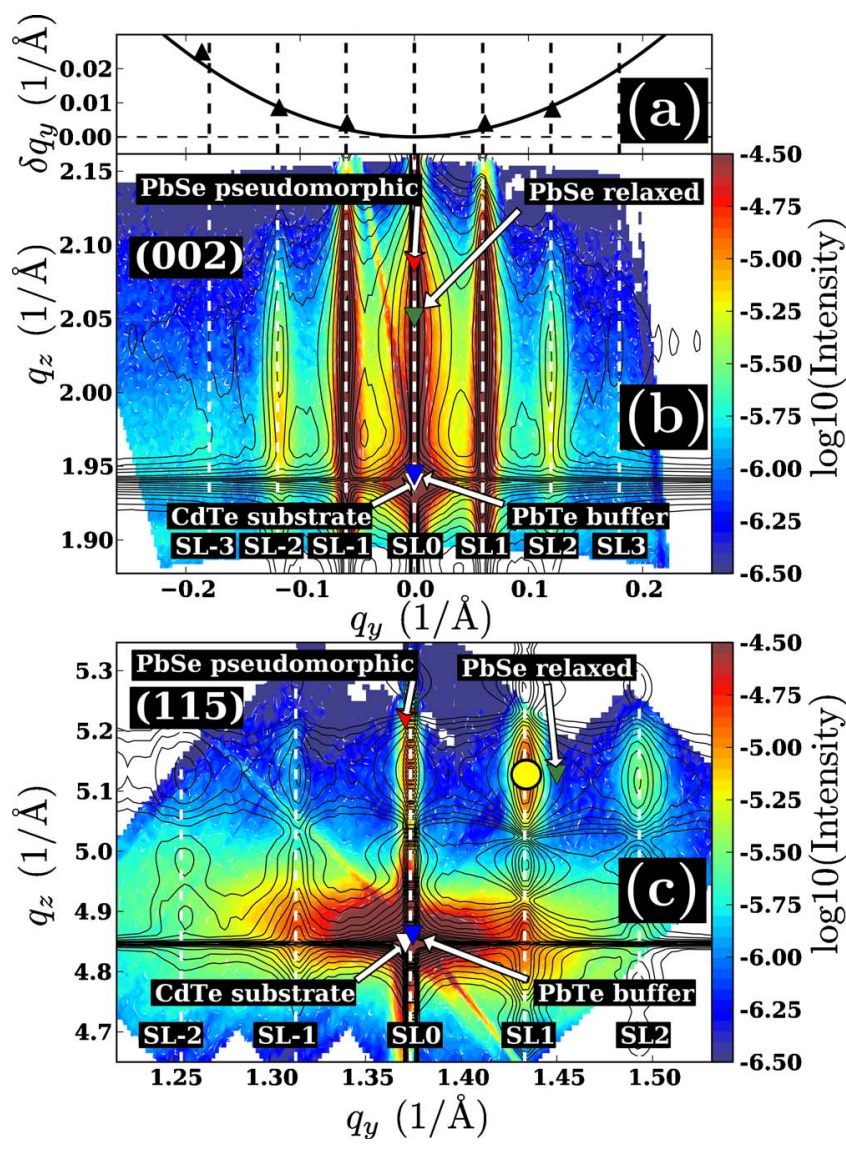

FIG. 1. (Color online) (a) shows the experimental (triangular symbols) widths vs position of the lateral satellites and the corresponding parabolic fit (black line) using Eq. (2). The (b) and (c) show the experimental (color plot) and simulated (black contour line) RSMs for the (002) and the (115) diffraction, respectively. The yellow spot in the (115) RSM denotes the Bragg peak of the PbSe epilayer. In the (002) map satellites up to the third order are visible while in the (115) map only second order satellites can be seen. The simulation data is from a dislocation ensemble with dislocation distances of $10.5 \pm 0.8 \mathrm{~nm}$.

In both RSMs shown in Fig. 1 the reflection from the PbSe epilayer breaks up into lateral satellites. The method is sufficiently sensitive to show satellites in the symmetric (002) RSM, though the diffraction vector and the Burgers vector are perpendicular to each other. Assuming that these lateral satellites are due to a periodic dislocation network, we obtain the mean distance of the dislocations from the distances $\Delta q_{y}$ between the oscillations with $\langle L\rangle=2 \pi / \Delta q_{y}$ $=10.5 \mathrm{~nm}$. The fact that this value is virtually equal to the one we obtained from the strain analysis supports our initial assumption of a periodic dislocation network.

For a further analysis of the satellites in the (002) RSM we obtained their positions $q_{y}$ and full width at half maximum values $\delta q_{y}$ by applying Gauss fits to each satellite and plotted $\delta q_{y}$ versus $q_{y}$ as shown in Fig. 1(a). Obviously $\delta q_{y}$ increases with satellite order.

The peak width $\delta q_{y}$ and position $q_{y}$ relative to the zeroth-order peak can be fitted by a parabolic function

$$
\delta q_{y}=\frac{\left(\sigma_{L} q_{y}\right)^{2}}{\langle L\rangle},
$$

which is a clear indication for short-range-ordering. ${ }^{12} \mathrm{We}$ obtained $\sigma_{L}=0.81 \mathrm{~nm}$, i.e., a mean deviation of dislocation distance from the average value of about $8 \%$.
To verify that the satellites and their broadening are really caused by dislocations and not by some other defects we compared our experimental data with computer simulations of the diffraction pattern around the (002) and (115) Bragg peaks. For the simulation of the scattered intensity we used kinematic approximation, where the scattered wave field can be written as ${ }^{13}$

$$
E(\mathbf{q}) \propto \int_{V} d \mathbf{r} \chi_{\mathbf{h}}(\mathbf{r}) e^{-i \mathbf{h u}(\mathbf{r})} e^{-i \mathbf{q r}} .
$$

$\chi_{\mathbf{h}}$ denotes the complex structure factor, $\mathbf{q}$ is the deviation of the momentum transfer between incident and diffracted wave $\mathbf{Q}$ from the substrate Bragg peak $\mathbf{h}$, which serves as a reference point; $\mathbf{Q}=\mathbf{h}+\mathbf{q}$, and $\mathbf{u}$ denotes the displacement field due to the dislocations, which was calculated by means of the extended FEM (XFEM) developed by Belytschko ${ }^{14}$ and first applied to dislocations by Gracie. ${ }^{15,16}$ Unlike analytical solutions, XFEM can handle anisotropic materials as well as complex dislocation geometries.

Typically, one would calculate the scattered intensity from the dislocation network as $I(\mathbf{q}) \propto\left\langle|E(\mathbf{q})|^{2}\right\rangle$, where \langle\rangle denotes averaging over all statistical quantities appearing in $|E(\mathbf{q})|^{2}$. In general this average cannot be evaluated analytically without simplifying assumptions. Furthermore, such an analytic approach is hardly useful in cases where the input data (here the displacement field) is calculated by means of a numerical method like XFEM. We have therefore chosen an alternative approach where the diffracted intensity is obtained as

$$
I(\mathbf{q}) \approx \frac{1}{N} \sum_{i=0}^{N-1}\left|E_{i}(\mathbf{q})\right|^{2} .
$$

Here the averaging is replaced by the incoherent summation over intensities scattered from different XFEM domains $\left|E_{i}(\mathbf{q})\right|^{2}$. Although being independent of each other, they share the same statistical properties. This is actually very similar to the physical situation, where the measured signal is the incoherent sum of the scattering of "patches" with a size determined by the lateral coherence length of the x-ray beam, typically of the order of micrometers. This approach has the advantage that it is entirely independent of the structures under investigation and therefore can be applied to a large number of different problems. For our simulations $N$ $=30$ domains were used for different values of $\sigma_{L}$ (namely, $0.75,0.8$, and $0.85 \mathrm{~nm}$ ). Each XFEM domain had a lateral size $210 \times 210 \mathrm{~nm}^{2}$ and a substrate thickness of $700 \mathrm{~nm}$. In reality, the coherent patch size is slightly larger than in the simulation, and $N \approx 10^{4}$ is much larger, but we noticed that in our case the incoherent sum shows no significant changes already for $N>20$.

Figure 2(a) shows a sketch of the domain model. The dislocations forming the network nucleate at the $\mathrm{PbTe} / \mathrm{PbSe}$ interface and run along the crystallographic [110] and [1 $\overline{1} 0]$ directions as sketched Fig. 2(a). They are of pure edge character with a Burgers vectors pointing in [ $\overline{1} \overline{1} 0](-y)$ direction for dislocations running along $[1 \overline{1} 0](x)$ direction and in [1]̄0] $(x)$ for dislocations running along [110] $(y)$ direction. Within a short range order model the probability of finding a certain distance $L$ of dislocations can be described by a normal- (Gauss-) distribution ${ }^{17}$ 


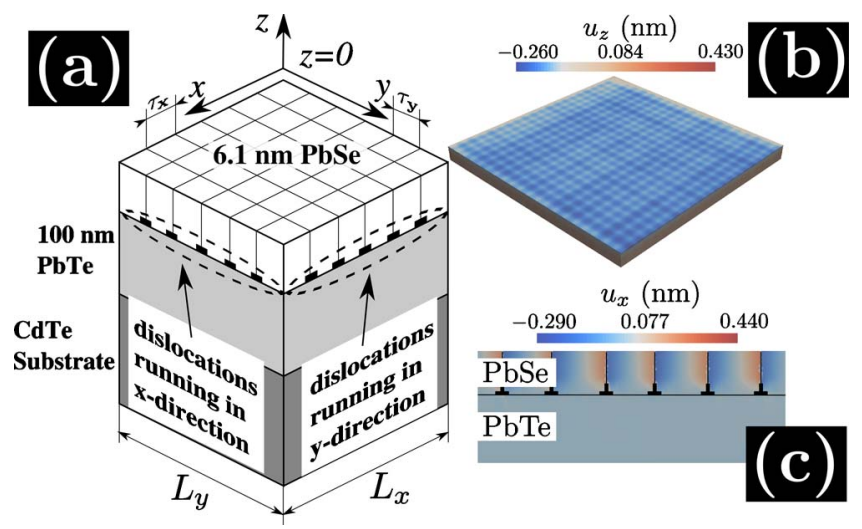

FIG. 2. (Color online) (a) shows the XFEM model for sample VA696a. The lateral size of the computational domain is $L_{x}=L_{y}=210 \mathrm{~nm}$. 20 dislocations were placed in $x$ - and $y$-direction, respectively. The $x$ - and $y$-axis run along the crystallographic [1힐 and [110] directions, respectively. The dislocations were placed randomly showing a Gaussian distance distribution [see Eq. (5)]. (b) shows the $u_{z}$ displacement for a single XFEM domain in the top layer. (c) shows a detailed view of the $u_{x}$ displacement for dislocations running in $y$-direction. Both figures on the right side are from an ensemble with $\sigma_{L}=0.8$.

$$
f(L)=\frac{1}{\sigma_{L} \sqrt{2 \pi}} \exp \left[-\frac{1}{2}\left(\frac{L-\langle L\rangle}{\sigma_{L}}\right)^{2}\right] .
$$

20 dislocations were placed randomly for each XFEM domain in each direction. Their positions were generated by a random number generator drawing random numbers following the Gaussian distribution above, with $\langle L\rangle$ taken from the experiment. Figures 2(b) and 2(c) show the $z$-displacement in the top-layer (top image) and a detailed view of the $u_{x}$ displacement due to dislocations running in $y$-direction for one of the domains with $\sigma_{L}=0.8 \mathrm{~nm}$. The results of the x-ray simulations for dislocations ensembles with $\sigma_{L}=0.8 \mathrm{~nm}$ are denoted by the black contour lines in the RSMs depicted in Figs. 1(b) and 1(c). The excellent match between the simulation and the experiment clearly proves that the strain field due to the dislocations gives rise to the satellite peaks. Moreover, in the simulation we assume that all dislocations have the same Burgers vector. If the actual dislocation network would contain dislocations with different Burgers vector the scattering pattern would be different. Hence, the simulation also allows to determine the dislocation's Burgers vector.

In conclusion, our combination of XFEM and XRD simulation using discrete ensemble averaging is well suited for the simulation of XRD from real from real dislocation networks. In the presented study, this allowed for quantitative analysis of the dislocation distribution in a $\mathrm{PbSe} / \mathrm{PbTe}$ epilayer system.

Work was supported by FWF, Vienna (Grant No. P18125000), EC, Brussels (Grant No. II-20060180 EC).

${ }^{1}$ Semiconductor Nanostructures, edited by D. Bimberg (Springer, New York, 2008).

${ }^{2}$ M. Ieong, B. Doris, J. Kedzierski, K. Rim, and M. Young, Science 306, 2057 (2004).

${ }^{3}$ J. Stangl, V. Holy, and G. Bauer, Rev. Mod. Phys. 76, 725 (2004).

${ }^{4}$ V. M. Kaganer, R. Köhler, M. Schmidbauer, R. Opitz, and B. Jenichen, Phys. Rev. B 55, 1793 (1997).

${ }^{5}$ V. Holý, T. Baumbach, D. Lübbert, L. Helfen, M. Ellyan, P. Mikulik, S. Keller, S. P. DenBaars, and J. Speck, Phys. Rev. B 77, 094102 (2008).

${ }^{6}$ V. M. Kaganer, O. Brandt, A. Trampert, and K. H. Ploog, Phys. Rev. B 72, 045423 (2005).

${ }^{7}$ S. Daniš, V. Holy, Z. Zhong, G. Bauer, and O. Ambacher, Appl. Phys. Lett. 85, 3065 (2004)

${ }^{8}$ D. K. Satapathy, V. M. Kaganer, B. Jenichen, W. Braun, L. Däweritz, and K. H. Ploog, Phys. Rev. B 72, 155303 (2005).

${ }^{9}$ V. Holý, J. H. Li, G. Bauer, F. Schäffler, and H.-J. Herzog, J. Appl. Phys. 78, 5013 (1995).

${ }^{10}$ G. Springholz and K. Wiesauer, Phys. Rev. Lett. 88, 015507 (2001).

${ }^{11}$ Y. H. Xie, G. H. Gilmer, C. Roland, P. J. Silverman, S. K. Buratto, J. Y. Cheng, E. A. Fitzgerald, A. R. Kortan, S. Schuppler, M. A. Marcus, and P. H. Citrin, Phys. Rev. Lett. 73, 3006 (1994).

${ }^{12}$ I. Kegel, T. H. Metzger, J. Peisl, J. Stangl, G. Bauer, and D. Smiglies, Phys. Rev. B 60, 2516 (1999).

${ }^{13} \mathrm{U}$. Pietsch, V. Holy, and T. Baumbach, High-Resolution X-Ray Scattering (Springer, New York, 2004).

${ }^{14}$ T. Belytschko and T. Black, Int. J. Numer. Methods Eng. 45, 601 (1999).

${ }^{15}$ R. Gracie, G. Ventura, and T. Belytschko, Int. J. Numer. Methods Eng. 69, 423 (2007).

${ }^{16}$ R. Gracie, J. Oswald, and T. Belytschko, J. Mech. Phys. Solids 56, 200 (2008).

${ }^{17}$ Since the dislocation distances can take only positive values a Poisson distribution would be more natural. However, since $\sigma_{L}$ in our case is much smaller then the mean distance $\langle L\rangle$ a Gaussian distribution gives virtually the same results. 